\title{
Does a laparoscopic approach attenuate the body weight loss and lean body mass loss observed in open distal gastrectomy for gastric cancer? a single-institution exploratory analysis of the JCOG 0912 phase III trial
}

\author{
Toru Aoyama ${ }^{1,2}$ - Tsutomu Sato ${ }^{1,2} \cdot$ Tsutomu Hayashi $^{1,2} \cdot$ Takanobu Yamada $^{1,2}$. \\ Haruhiko $\mathrm{Cho}^{1} \cdot$ Takashi Ogata $^{1} \cdot \mathrm{Koji} \mathrm{Oba}^{3} \cdot$ Takaki Yoshikawa $^{1,2}$
}

Received: 21 March 2017/Accepted: 2 June 2017/Published online: 16 June 2017

(C) The International Gastric Cancer Association and The Japanese Gastric Cancer Association 2017

\begin{abstract}
Background Laparoscopy-assisted distal gastrectomy (LADG) for gastric cancer may prevent the loss of body weight and lean body mass resulting from reduced surgical stress in comparison to open distal gastrectomy (ODG). A multicenter phase III trial conducted by the Japan Clinical Oncology Group (JCOG0912 trial) was performed to confirm the non-inferiority of LADG to ODG for stage I gastric cancer in terms of relapse-free survival.

Methods This study was performed as a single-institution exploratory analysis using the data of the patients from our hospital who were enrolled in the JCOG0912 phase III trial. Body weight and lean body mass were evaluated using a bioelectrical impedance analyzer within 1 week before and at 1 week, 1 month, and 3 months after surgery.

Results One-hundred six patients were randomized to undergo ODG (54 patients) or LADG (51 patients). Body weight loss at 1 week, 1 month, and 3 months was $-3.0 \%$, $-4.9 \%$, and $-5.4 \%$, respectively, in the ODG group and $-2.7 \%,-4.3 \%$, and $-5.7 \%$, respectively, in the LADG group; the differences were not statistically significant ( $p=0.330,0.166$, and 0.656 , respectively). Lean body mass loss at 1 week, 1 month, and 3 months was $-2.8 \%$,
\end{abstract}

Toru Aoyama and Takaki Yoshikawa contributed equally to this article.

Takaki Yoshikawa

yoshikawat@kcch.jp

1 Department of Gastrointestinal Surgery, Kanagawa Cancer Center, 2-3-2 Nakao, Asahi-ku, Yokohama 241-8515, Japan

2 Department of Surgery, Yokohama City University, Yokohama, Japan

3 Department of Biostatistics, The University of Tokyo, Tokyo, Japan
$-4.1 \%$, and $-2.3 \%$, respectively, in the ODG group and $-2.7 \%,-2.9 \%$, and $-3.0 \%$, respectively, in the LADG group; the differences were not statistically significant ( $p=0.610,0.413$, and 0.925 , respectively).

Conclusions The laparoscopic approach did not attenuate the loss of body weight and lean body mass in comparison to patients who underwent open distal gastrectomy for gastric cancer.

Keywords Body composition - Lean body mass . Laparoscopy-assisted distal gastrectomy · Open distal gastrectomy $\cdot$ Randomized

\section{Introduction}

The use of laparoscopy-assisted distal gastrectomy (LADG) to treat gastric cancer was first described by Kitano in 1994 [1]. Since then, the number of cases of gastric cancer treated with LADG has gradually increased. The advantages of this procedure in comparison to open distal gastrectomy (ODG) include reduced amount of operative blood loss, reduced pain, earlier recovery of bowel activity and resumption of oral intake, and shorter hospital stay [2,3]. Moreover, some reports have suggested that LADG is associated with lower levels of surgical stress and a lower incidence of impaired nutrition in comparison to ODG [4]. With regard to survival, the Japan Clinical Oncology Group (JCOG) conducted a multicenter phase III trial to confirm the non-inferiority of LADG to ODG for stage I gastric cancer in terms of relapsefree survival (JCOG0912) [5].

When surgical stress occurs, the immune cells produce cytokines that act as mediators of both immune and systemic responses to injury [6]. Muscle catabolism is accelerated by the cytokines that are produced during and after surgery, 
which leads to a decrease in muscle content after surgery. Gastric cancer surgery, which is a category of major abdominal surgery, is associated with loss of body weight and loss of lean body mass. We recently clarified that postoperative body weight and lean body mass losses were significant risk factors for compliance to adjuvant chemotherapy and were related to chemotherapy-induced toxicities in patients with gastric cancer [7, 8]. More recently, we demonstrated that postoperative body weight loss was a significant prognostic factor for survival in gastric cancer patients. Theoretically, less invasive surgery attenuates muscle catabolism. If less invasive surgery can attenuate the loss of body weight and of lean body mass, then compliance to adjuvant chemotherapy could be increased and the prognosis could be improved. To confirm this, we compared the results of LADG and ODG in randomized patients who received the same perioperative care.

The present study aimed to clarify whether LADG attenuates the loss of body weight and lean body mass after gastrectomy in a single-institutional exploratory analysis of the JCOG0912 phase III trial. All the patients received the same perioperative care via a fast-track surgery protocol in the Kanagawa Cancer Center.

\section{Patients and methods}

\section{Patients}

This study was performed as a single-institution exploratory analysis of the Japan Clinical Oncology Group (JCOG)-0912 trial. The JCOG-0912 trial was a multicenter phase III trial to confirm the non-inferiority of LADG to ODG in terms of relapse-free survival in patients with clinical stage I gastric cancer disease who were diagnosed according to the 14th edition of the general rules for gastric cancer published by the Japanese Gastric Cancer Association (UMIN-ID 000003319) [9, 10]. The details of the JCOG-0912 trial have been previously reported [11]. The accrual of patients for the JCOG-0912 trial was initiated in March 2010 and terminated in November 2013. Institutions were selected as a stratification factor for randomization in the JCOG-0912 trial.

\section{Surgical procedures}

All the patients underwent distal gastrectomy with nodal dissection. Regardless of the surgical approach, D1+ nodal dissection was applied for clinical stage IA tumors and D2 dissection was applied for clinical stage IB tumors.

In accordance with the protocol of the JCOG-0912 trial, one of two certified laparoscopic staff surgeons was responsible for the surgical quality of laparoscopic surgery.
Five or six ports were used. Lymph node dissection was performed in the laparoscopic field. The omentum was preserved except where resection was necessary for lymph node dissection along the right gastroepiploic artery. A small abdominal incision $(\leq 6 \mathrm{~cm})$ was made in the upper abdomen for the removal of the specimen and reconstruction. Reconstruction was principally performed using Billroth-I gastroduodenostomy, and all the reconstruction procedures were performed extracorporeally using circular staplers [12]. For open surgery, an upper abdominal median incision extending from the xiphoid to the navel was created. The nodal dissection and reconstruction procedure was the same as that used in the laparoscopic approach. A circular stapler of $25-\mathrm{mm}$ diameter was used principally for BI anastomosis. In addition, no drain was used for either laparoscopy or open distal gastrectomy. If the surgeon thought that drain placement was necessary because of postoperative bleeding or pancreatitis, a low-vacuum drainage system was left in the subhepatic area for peritoneal fluid collection.

\section{Perioperative care}

The patients received the same enhanced recovery after surgery (ERAS) protocol after both ODG and LADG. The details of the ERAS protocol have been previously reported [13]. In brief, the patients were allowed to eat until midnight on the day before surgery and were required to drink the contents of two 500-ml plastic bottles containing an oral rehydration solution until $3 \mathrm{~h}$ before surgery. The nasogastric tube was removed immediately after surgery. Oral intake was initiated on postoperative day (POD) 2, beginning with water and an oral nutritional supplement. The patients began to eat solid food on POD 3, starting with rice gruel and soft food on POD 3, and advancing in three steps to regular food intake on POD 7. The patients were discharged on POD 7 when they had achieved adequate pain relief and soft food intake, had returned to their preoperative mobility level, and exhibited normal laboratory data.

\section{Evaluation of operative morbidity and mortality}

Surgical and nonsurgical complications were assessed prospectively and were classified according to the ClavienDindo classification [14]. Operative mortality was defined as postoperative death from any cause within 30 days after surgery or during the same hospital stay.

\section{Body composition analysis}

The segmental body composition was analyzed using an MC-190EM bioelectrical impedance analyzer (Tanita, 
Fig. 1 Consort diagram of the present study

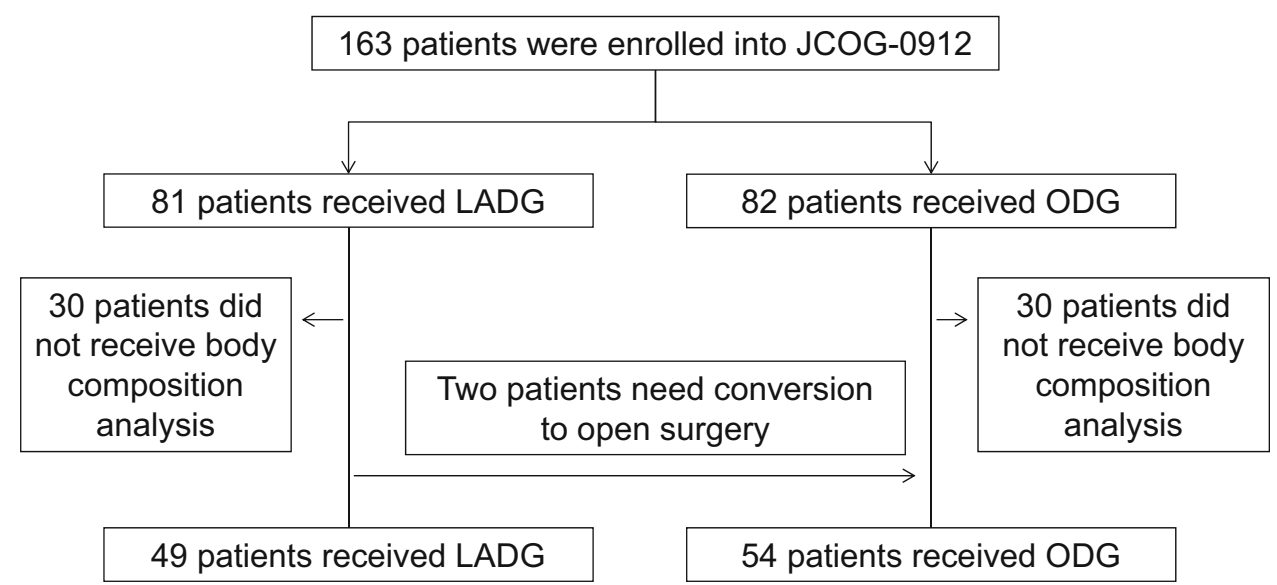

Tokyo, Japan), which provides relative information regarding the amount of lean tissue and fat tissue in the trunk area and each limb, as well as the overall body composition and hydration status. Body weight and composition were evaluated by the bioelectrical impedance analyzer within 1 week before surgery and at 1 week, 1 month, and 3 months after surgery. The time for body composition analyses was morning before breakfast before and 1 week after surgery, whereas that timing was not determined at 1 and 3 months after surgery.

\section{Evaluations, statistical analyses, and ethical considerations}

Body weight $(\mathrm{BW})$ loss was defined as $\% \mathrm{BW}$ loss $=$ (preoperative BW-BW at 1 week, 1 month, and 3 months after surgery) $\times 100$ /preoperative body weight. Lean body mass (LBM) loss was defined as \% LBM loss = (preoperative LBM-LBM at 1 week, 1 month, and 3 months after surgery) $\times 100 /$ preoperative LBM. The values are expressed as the median and range. The data were compared using the chisquared test and the Wilcoxon signed-rank test. $p$ values $<0.05$ were considered to indicate statistical significance. The SPSS software program (version 12.0 J Win; SPSS, Chicago, IL, USA) was used for all statistical analyses. The R category and extent of dissection were determined according to the Japanese Classification of Gastric Carcinoma, third English edition, and the Japanese Gastric Cancer Association guidelines [10]. The institutional review board of our hospital approved the JCOG-0912 phase III study and this exploratory analysis. The primary investigators of the JCOG-0912 trial, the representative director of the JCOG Gastric Cancer Study Group, and the chairperson of the JCOG approved this exploratory study of the JCOG-0912 trial. All data were prospectively collected.

\section{Results}

\section{Background}

A total of 163 patients who were treated at the Kanagawa Cancer Center were enrolled in the JCOG-0912 study. Among them, 103 consecutive patients were examined in this study. The initial 60 patients, who were enrolled from April 2010 to April 2011, were excluded from this study because their body composition was not measured. A flow diagram of the study is shown in Fig. 1. Fifty-two patients underwent ODG and 51 patients underwent LADG. Conversion to open surgery was necessary in $2(1.9 \%)$ patients because of bleeding; these patients were included in the ODG group. The background characteristics and baseline data were well randomized within both groups (Table 1). No patients had any history of weight loss, appetite loss, or decrease in food intake before surgery.

\section{Surgical and pathological outcomes}

Two patients who were assigned to undergo LADG were converted to open surgery. Duration of surgery in the LADG group was significantly longer than that in the ODG group $(p<0.001)$. However, the amount of bleeding in the LADG group was significantly lower than that in the ODG group $(p=0.003)$ (Table 2). No differences were observed in the pathological outcomes of the two groups.

No mortalities occurred in either group. The surgical morbidities are shown in Table 3. The incidence of the surgical complications was similar in the two groups.

Median hospital stay was 9 days (range, 6-76 days) in the ODG group and 9 days (range, 7-18 days) in the LADG group. There was no statistical difference between the two groups. 
Table 1 Comparison of the patient background

\begin{tabular}{|c|c|c|c|}
\hline & ODG $[n=54,(\%)]$ & LADG $[n=49,(\%)]$ & $p$ value \\
\hline $\begin{array}{l}\text { Age, years (median) } \\
\text { (range) }\end{array}$ & $\begin{array}{l}68.5 \\
(36-78)\end{array}$ & $\begin{array}{l}64 \\
(33-79)\end{array}$ & 0.422 \\
\hline Gender & & & 0.855 \\
\hline Male & $31(57.4 \%)$ & $29(59.2 \%)$ & \\
\hline Female & $23(42.6 \%)$ & $20(40.8 \%)$ & \\
\hline ASA-PS & & & 0.565 \\
\hline 1 & $15(27.8 \%)$ & $15(30.6 \%)$ & \\
\hline 2 & $39(72.2 \%)$ & $33(67.2 \%)$ & \\
\hline 3 & $0(0 \%)$ & $1(2 \%)$ & \\
\hline $\begin{array}{l}\text { Total body weight, kg (median) } \\
\text { (range) }\end{array}$ & $\begin{array}{l}56.1 \\
(39.2-85.5)\end{array}$ & $\begin{array}{l}59.4 \\
(35.8-77.5)\end{array}$ & 0.978 \\
\hline $\begin{array}{l}\text { Lean body mass, kg (median) } \\
\text { (range) }\end{array}$ & $\begin{array}{l}42.2 \\
(29.1-60.6)\end{array}$ & $\begin{array}{l}44.4 \\
(29.4-58.3)\end{array}$ & 0.991 \\
\hline Tumor site & & & 0.323 \\
\hline Body & $30(55.6 \%)$ & $27(55.1 \%)$ & \\
\hline Antrum & $24(44.4 \%)$ & $22(44.9 \%)$ & \\
\hline Clinical $\mathrm{T}$ factor & & & 0.404 \\
\hline $\mathrm{T} 1$ & $38(70.4 \%)$ & $36(73.5 \%)$ & \\
\hline $\mathrm{T} 2$ & $16(29.6 \%)$ & $13(26.5 \%)$ & \\
\hline Clinical $\mathrm{N}$ factor & & & 0.277 \\
\hline Negative & $51(94.4 \%)$ & $49(100 \%)$ & \\
\hline Positive & $3(5.6 \%)$ & $0(0 \%)$ & \\
\hline \multicolumn{4}{|l|}{ Co-morbidity } \\
\hline Hypertension & $20(37.0 \%)$ & $21(42.9 \%)$ & 0.420 \\
\hline Diabetes mellitus & $4(7.4 \%)$ & $4(8.2 \%)$ & 0.886 \\
\hline COPD & $7(13.0 \%)$ & $4(8.2 \%)$ & 0.529 \\
\hline
\end{tabular}

$A S A-P S$ ASA physical status, $C O P D$ chronic obstructive pulmonary disease

\section{Body composition changes}

The median \% BW loss (range) was $-2.7 \%$ ( -7.2 to 1.6$)$ at 1 week after surgery, $-4.3 \%$ ( -9.3 to 3.5$)$ at 1 month after surgery, and $-5.7 \%$ ( -11.7 to 5.1$)$ at 3 months after surgery in the LADG group, and $-3.0 \%(8.6-2.7)$ at 1 week after surgery, $-4.9 \%(-15.4$ to -0.2$)$ at 1 month after surgery, and $-5.4 \%$ ( -22.0 to 0.9$)$ at 3 months after surgery in the ODG group (Fig. 2). No significant differences were observed in the \% BW loss of the two groups at any of the time points ( $p=0.330, p=0.166$, and $p=0.656$, respectively). Moreover, the median \% LBM loss (range) was $-2.7 \%$ ( -7.7 to 6.1$)$ at 1 week after surgery, $-2.9 \%(-11.4$ to 9.3$)$ at 1 month after surgery, and $-3.0 \%(-9.1$ to 7.7$)$ at 3 months after surgery in the LADG group, and $-2.8 \%$ $(-13.0$ to 6.7$)$ at 1 week after surgery, $-4.1 \%(-20.4$ to 9.8$)$ at 1 month after surgery, and $-2.3 \%$ ( -16.9 to 9.3$)$ at 3 months after surgery in the LADG group (Fig. 3). No significant differences were observed in the \% LBM loss of the two groups at any of the time points $(p=0.610$, $p=0.413$, and $p=0.925$, respectively). The response rate of the measurement in the LADG group was $100 \%(49 / 49)$ before and at 1 week after surgery, 93.9\% (46/49) at 1 month after surgery, and $83.7 \%(41 / 49)$ at 3 months after surgery; that in the ODG group was $100 \%(54 / 54)$ before and at 1 week after surgery, $94.4 \%$ (51/54) at 1 month after surgery, and $88.9 \%(48 / 54)$ at 3 months after surgery.

\section{Discussion}

This is the first study to compare the loss of body weight and lean body mass in patients who received LADG and ODG in a randomized cohort at a single institution. Very recently, Matsushita et al. reported that body composition and nutritional status during 1 year after distal gastrectomy were similar between the laparoscopic and open approach. Although their study was a prospective multicenter study with preplanned sample size and the background characteristics were similar, the use of laparoscopic or open approach was not randomly allocated but was determined by the physician's choice [15]. Therefore, selection bias could induce deviation 
Table 2 Surgical and pathological outcomes

\begin{tabular}{llll}
\hline & ODG $(n=54)$ & LADG $(n=49)$ & $p$ value \\
\hline Lymph node dissection & & & 0.944 \\
D1+ dissection & $38(70.4 \%)$ & $36(73.5 \%)$ & \\
D2 dissection & $16(29.6 \%)$ & $13(26.5 \%)$ & 0.450 \\
Reconstruction & & & \\
Billroth-1 & $47(87.0 \%)$ & $40(81.6 \%)$ & 0.003 \\
Roux-en-Y & $7(13.0 \%)$ & $9(18.4 \%)$ & $<0.001$ \\
Bleeding, g (median) & 130 & 50 & \\
$\quad$ (range) & $(20-1400)$ & $(5-530)$ & 0.967 \\
Operation time, min (median) & 179 & 290 & \\
(range) & $(95-492)$ & 42 & 0.357 \\
Number of harvested lymph nodes & 43 & $(15-83)$ & \\
(median, range) & $(14-114)$ & $40(81.6 \%)$ & \\
Pathological T factor & & $9(18.4 \%)$ & 0.204 \\
T0-T1 & $44(81.5 \%)$ & $44(89.8 \%)$ & \\
T2- & $10(18.5 \%)$ & $5(10.2 \%)$ & \\
Pathological N factor & & & \\
N0 & $48(88.9 \%)$ & $6(11.1 \%)$ & \\
N1- & & & \\
\hline
\end{tabular}

\begin{tabular}{llll}
\hline & ODG $[\mathrm{n}=54,(\%)]$ & LADG $[n=49,(\%)]$ & $p$ value \\
\hline Total & $5(9.3 \%)$ & $4(8.2 \%)$ & 0.327 \\
Pancreatic fistula & Grade $2: 1$ & Grade 2: 1 & \\
& & Grade 3a: 1 & \\
Anastomotic leakage & Grade 2:1 & 0 \\
& Grade 3a: 1 & \\
Abdominal abscess & Grade 3a: 1 & 0 \\
Pneumonia & 0 & Grade 2: 1 \\
Anastomotic stenosis & Grade 3b: 1 & 0 & \\
Postoperative bleeding & 0 & Grade 2: 1 & \\
\hline
\end{tabular}

Table 3 Comparison of morbidity and mortality between the ODG and LADG groups of unknown background factors between the two groups, which may affect their results. Moreover, perioperative care was not standardized in their study. The present study clearly demonstrated that the loss of body weight and lean body mass in the LADG and ODG groups did not differ to a statistically significant extent in the randomized comparison of laparoscopic and open approach with the same perioperative care via a fast-track surgery protocol.

There are several possible reasons why the loss of body weight and lean body mass was similar between the LADG and ODG groups. The first major reason must be that the intraabdominal surgical procedures, the resection of more than two thirds of the stomach with lymph node dissection, were the same between open and laparoscopic gastrectomy. Surgical stress may be determined not by the length of the skin incision but by the intraabdominal surgical procedures. The second possible reason is that both procedures might have involved a similar degree of surgical stress. We previously investigated the surgical stress of patients who were randomized to undergo ODG $(n=13)$ and LADG $(n=13)$. All the patients received the same perioperative care via a fast-track surgery protocol [16]. In that study, we found that the level of surgical stress was similar in the ODG and LADG groups. Similar results were observed in the field of colon cancer surgery [17, 18]. On the other hand, Hayashi et al. evaluated the levels of interleukin-6 (IL-6) and C-reactive protein (CRP) and the whole blood cell (WBC) counts in patients who underwent LADG $(n=14)$ and ODG $(n=14)$ in a randomized study, and demonstrated that the degree of surgical stress among the patients who underwent ODG was significantly higher than that among patients who underwent LADG [19]. Thus, it remains controversial whether laparoscopic surgery is actually associated with less surgical stress than open surgery. The third possibility is that the loss of body weight and lean body mass is not caused only by surgical stress but 
Fig. 2 Changes in the percent (\%) body weight loss in the laparoscopy-assisted distal gastrectomy group $(L A D G)$ and the open distal gastrectomy group $(O D G)$
Fig. 3 Changes in the percent (\%) lean body mass loss in the laparoscopy-assisted distal gastrectomy group and the open distal gastrectomy group

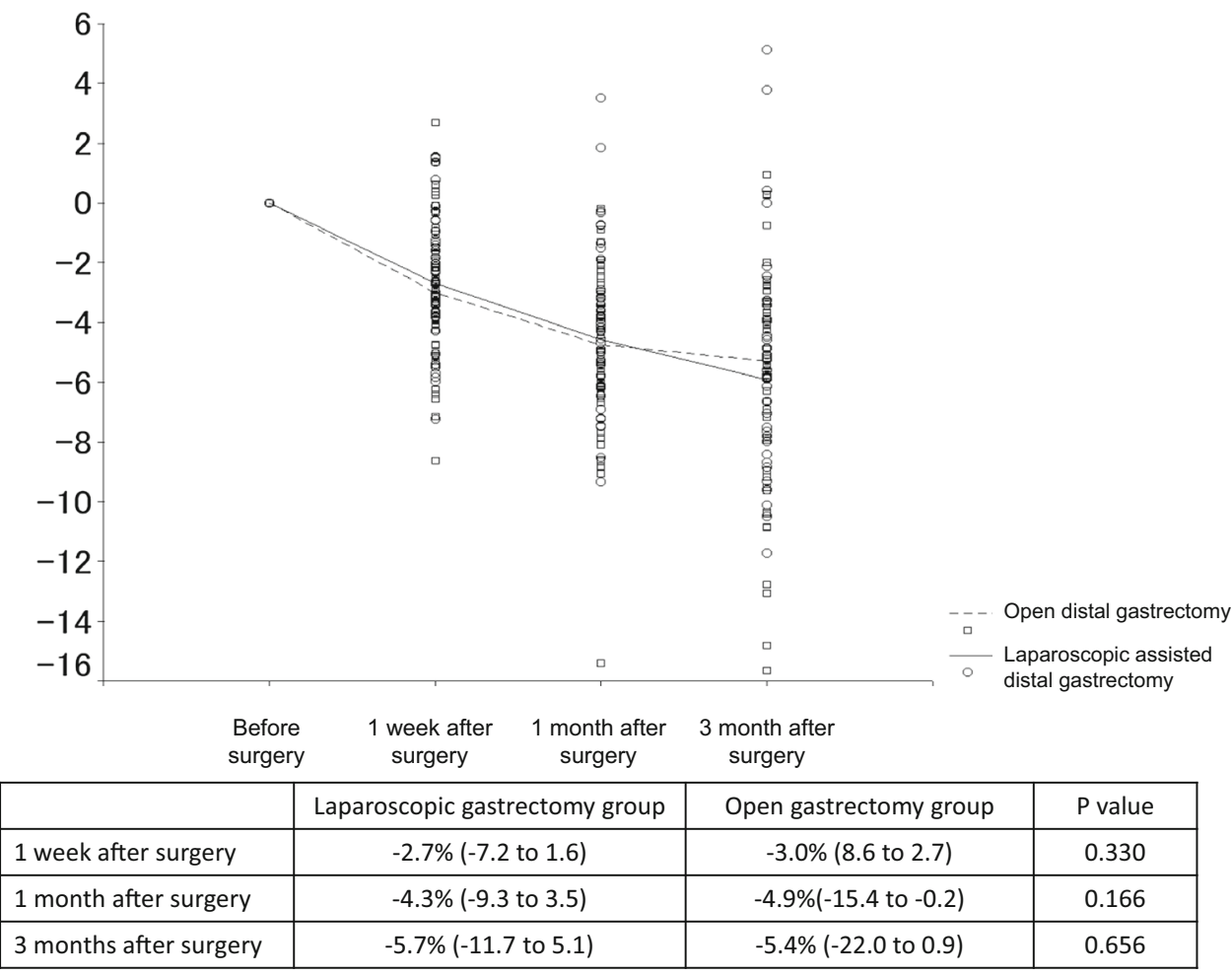

also by the loss of food intake after gastrectomy. Most patients of both groups received Billroth-1 reconstruction. Both groups received similar reconstruction using the same circular stapler extracorporeally. Although we could not know the exact calorie intake after surgery, it is likely that both groups had a similar loss of calorie intake after surgery, which might have been associated with the loss of body weight and lean body mass. 
The background characteristics and operative details of the patients in the present study were similar to those of the patients in the JCOG-0912 trial [11]. Moreover, the incidence and details of postoperative surgical complications in the present study were similar to those of the JCOG0912 trial. Grade $\geq 3$ surgical complications were observed in $3.9 \%(4 / 103)$ of the patients in the current study and in $3.5 \%(32 / 912)$ of the patients in the JCOG-0912 trial. In addition, the rates of grade $\geq 3$ pancreatic fistula $(0.9 \%$ vs. $0.4 \%$ ), anatomic leakage ( $0.9 \%$ vs. $0.2 \%$ ), and abdominal abscess $(0.9 \%$ vs. $1.6 \%)$ in the present study and the JCOG-0912 trial were similar. Thus, our randomized cohort had characteristics similar to those of the cohort of the JCOG-0912 trial, which suggested that the present results were not specific in our randomized cohort but a general finding.

In the present study, there were some limitations. First, the segmental body composition was analyzed using a bioelectrical impedance analyzer, which could not directly measure the muscle mass. The lean body mass is calculated from the sum of the muscle mass and the mass of the visceral organs. However, the mass of visceral organs would not be changed by surgery; thus, the major contributor to the change in the lean body mass would be a change in the muscle mass. Although the absolute muscle mass was not measured in this study, the comparability would have been maintained in this randomized cohort. Because the difference in body weight and lean body mass between the ODG and LADG groups was minimal, it is unlikely that the present results would be contradicted by a more accurate method. Second, the body composition was not measured at the same time. In the present study, the body composition was measured at the morning before breakfast before and 1 week after surgery, although the time for measurement was not pre-specified at 1 and 3 months after surgery. This difference was also a limitation of the present study because body water is influenced by oral intake.

In conclusion, the body composition changes in the ODG and LADG groups were similar in a randomized cohort from a single institution who received the same perioperative care via a fast-track surgery protocol. The results indicate that a laparoscopic approach does not attenuate the loss of body weight and lean body mass in comparison to open distal gastrectomy for gastric cancer.

\footnotetext{
Acknowledgments We thank Dr. Hitoshi Katai, a primary investigator in the JCOG-0912 trial, Professor Mitsuru Sasako, a past-representative leader of the Gastric Cancer Treatment Group of the JCOG, Dr. Masanori Terashima, a current representative leader of the Gastric Cancer Treatment Group of the JCOG, and Dr. Haruhiko Fukuda, the chairman of the JCOG. This work was supported, in part, by three nongovernmental organizations: the Kanagawa Standard Anti-cancer Therapy Support System, the Uehara Memorial
}

Foundation, and the Takeda Medical Foundation. The authors express their sincere gratitude to Ms. Yuka Fujita, Ms. Natsumi Sato, and Ms. Rika Takahashi for their excellent data management in this study.

\section{Compliance with ethical standards}

Conflict of interest statement The authors declare no conflicts of interest in association with the present study.

Human rights statement The study data and informed consent were obtained in accordance with the Declaration of Helsinki and were approved by the Ethics Review Board of Kanagawa Cancer Center.

Informed consent Informed consent or substitute for it was obtained from all patients for being included in the study.

\section{References}

1. Kitano S, Iso Y, Moriyama M, et al. Laparoscopy-assisted Billroth-I gastrectomy. Surg Laparosc Endosc. 1994;4:146-8.

2. Yakoub D, Athanasiou T, Tekkis P, et al. Laparoscopic assisted distal gastrectomy for early gastric cancer: is it an alternative to the open approach? Surg Oncol. 2009;18:322-33.

3. Zeng YK, Yang ZL, Peng JS, et al. Laparoscopy-assisted versus open distal gastrectomy for early gastric cancer: evidence from randomized and nonrandomized clinical trials. Ann Surg. 2012;256:39-52.

4. Adachi Y, Shiraishi N, Shiromizu A, et al. Laparoscopy-assisted Billroth I gastrectomy compared with conventional open gastrectomy. Arch Surg. 2000;135:806-10.

5. Nakamura K, Katai H, Mizusawa J, et al. A phase III study of laparoscopy-assisted versus open distal gastrectomy with nodal dissection for clinical stage IA/IB gastric cancer (JCOG0912). Jpn J Clin Oncol. 2013;43:324-7.

6. Castell JV, Gómez-Lechón MJ, David M, et al. Interleukin-6 is the major regulator of acute phase protein synthesis in adult human hepatocytes. FEBS Lett. 1989;242:237-9.

7. Aoyama T, Yoshikawa T, Shirai J, Hayashi T, Yamada T, Tsuchida $\mathrm{K}$, et al. Body weight loss after surgery is an independent risk factor for continuation of S-1 adjuvant chemotherapy for gastric cancer. Ann Surg Oncol. 2013;20:2000-6.

8. Aoyama T, Kawabe T, Fujikawa H, et al. Loss of lean body mass as an independent risk factor for continuation of S-1 adjuvant chemotherapy for gastric cancer. Ann Surg Oncol. 2015;22:2560-6.

9. Japanese Gastric Cancer Association. Japanese gastric cancer treatment guidelines 2010 (ver. 3). Gastric Cancer. 2011;14:113-23.

10. Japanese Gastric Cancer Association. Japanese classification of gastric carcinoma: 3rd English edition. Gastric Cancer. 2011;14:101-12.

11. Katai H, Mizusawa J, Katayama H, et al. Short-term surgical outcomes from a phase III study of laparoscopy-assisted versus open distal gastrectomy with nodal dissection for clinical stage IA/IB gastric cancer: Japan Clinical Oncology Group Study JCOG0912. Gastric Cancer 2016. [Epub ahead of print] PubMed PMID: 27718137.

12. Yoshikawa T, Cho H, Rino Y, et al. A prospective feasibility and safety study of laparoscopy-assisted distal gastrectomy for clinical stage I gastric cancer initiated by surgeons with much experience of open gastrectomy and laparoscopic surgery. Gastric Cancer. 2013;16:126-32.

13. Yamada T, Hayashi T, Cho H, et al. Usefulness of enhanced recovery after surgery protocol as compared with conventional 
perioperative care in gastric surgery. Gastric Cancer. 2012;15:34-41.

14. Clavien PA, Barkun J, de Oliveira ML, et al. The Clavien-Dindo classification of surgical complications: five-year experience. Ann Surg. 2009;250:187-96.

15. Matsushita $\mathrm{H}$, Tanaka $\mathrm{C}$, Murotani $\mathrm{K}$, Misawa $\mathrm{K}$, Ito $\mathrm{S}$, Ito $\mathrm{Y}$, et al. Nutritional recovery after open and laparoscopic distal gastrectomy for early gastric cancer: a prospective multicenter comparative trial (CCOG1204). Dig Surg. 2017;. doi:10.1159/ 000458714

16. Aoyama T, Yoshikawa T, Hayashi T, et al. Randomized comparison of surgical stress and the nutritional status between laparoscopy-assisted and open distal gastrectomy for gastric cancer. Ann Surg Oncol. 2014;21:1983-90.

17. Tang CL, Eu KW, Tai BC, Soh JG, MacHin D, Seow-Choen F. Randomized clinical trial of the effect of open versus laparoscopically assisted colectomy on systemic immunity in patients with colorectal cancer. Br J Surg. 2001;88:801-7.

18. Dunker MS, Ten Hove T, Bemelman WA, Slors JF, Gouma DJ, Van Deventer SJ. Interleukin-6, C-reactive protein, and expression of human leukocyte antigen-DR on peripheral blood mononuclear cells in patients after laparoscopic vs. conventional bowel resection: a randomized study. Dis Colon Rectum. 2003;46:1238-44.

19. Hayashi H, Ochiai T, Shimada H, et al. Prospective randomized study of open versus laparoscopy-assisted distal gastrectomy with extraperigastric lymph node dissection for early gastric cancer. Surg Endosc. 2005;19:1172-6. 